\title{
Attitudes towards shared decision making in mental health: A qualitative synthesis
}

\begin{abstract}
Purpose

Shared Decision Making (SDM) prioritises joint deliberation between practitioner and service user, and a respect for service-users' experiential knowledge, values and preferences.. This article reviews the existing literature pertaining to key stakeholders' attitudes towards SDM in mental health. It examines whether perceived barriers and facilitators differ by group (e.g. service user, psychiatrist, nurse, social worker) and includes views of what facilitates and hinders the process for service users and practitioners.
\end{abstract}

\section{Methodology}

This review adopts the principles of a qualitative research synthesis. A key word search of research published between 1990 and 2016 was undertaken. Qualitative, quantitative and mixed methods studies were included.

\section{Findings}

43 papers were included and several themes identified for service user and practitioner perspectives. Both practitioners and service users see SDM as an ethical imperative, and both groups highlight the need to be flexible in implementing shared decision making, suggesting it is context dependent. A range of challenges and barriers are presented by both practitioners and service users reflecting complex contextual and cultural features within which interactions in mental health take place. There were qualitative differences in what service users and practitioners describe as preventing or enabling SDM. The differences highlighted point towards different challenges and priorities in SDM for service users and practitioners.

\section{Originality / Value}

The presentation of nuanced views and attitudes that practitioners and service users hold represent an important and under reported area and offer insight into the reasons for the gap between idealised policy and actual practice of SDM in mental health settings.

Keywords: Shared Decision Making (SDM), Mental Health, Service Users perspectives, Practitioner perspectives, Qualitative Synthesis 


\section{Introduction}

Shared Decision Making (SDM) is endorsed as an essential component of everyday mental health practice delivery across much of the western and developed world. For some countries (e.g. areas of Scandanavia) patient involvement is seen as a right and enshrined in law (see Coulter et al, 2008). The increasing emphasis within policy on embedding principles of SDM in everyday psychiatric encounters represents broader change in the mental health system. As discussed in James and Quirk's paper in this issue, SDM represents a broader movement towards patient centred care and the increasing neo liberalisation of health care services. For mental health services, SDM is also seen as a central tool for models of personal recovery and recovery based practice in mental health (Schauer et al., 2007). Deegan and colleagues assert that it is time to "heed the ethical imperative upon which the practice of SDM rests" (Deegan and Drake, 2015, p 1008). Accordingly then, SDM represents a broader shift in mental health services towards giving mental health service users choice, enhanced control, and placing increased priority on partnership with practitioners as part of a personalised approach. Here the service providers' role is to assist in the process of individual led recovery and the corresponding change in the service users' re-evaluation of identity (Shepherd, Boardman and Slade, 2008). In this sense models of SDM represent an emphasis on a rebalancing of power in mental health encounters, and allowing for experiential and medical expertise to be equally respected and valued in decisions concerning treatment (Kaminskiy, 2015). Furthermore, SDM reflects the values of respect, enshrined in the Convention of the Rights of Persons with Disabilities (CRPD) by the United Nations and could be a way to prevent some situations that are directly criticised in the CRPD (e.g. coercive practice and forced medication use).

Nevertheless, SDM has emerged from other health care arenas. It occupies the middle of a spectrum of patient participation, between paternalism on the one end (derived from the biomedical paradigm of health where the doctor makes the decision after evaluation of the diagnosis, treatment options, and risks and benefits) and the informed model of decision-making on the other (which assumes that the service-user has ultimate responsibility for the decision made and the professional acts as an adviser) (Charles et al., 1999). Within SDM, priority is placed on communication and shared deliberation with a respect for service-users' experiential knowledge, values and preferences (Edwards and Elwyn, 2009; Makoul and Clayman, 2006).

While practitioners and service users alike generally endorse SDM (as we will outline in this paper), there is growing evidence that it is not implemented in everyday practice (e.g. see Ramon et al. in this issue). To better understand this gap it is necessary to further explore the nuanced views and attitudes that practitioners and service users hold. In addition, reviews with a focus on examining broader exploration of the perspectives and views about SDM among key stakeholders groups is scarce, and represent an important and under reported area. Legare et al's systematic review of health professionals' perceptions of SDM in 2008, and the later selective literature review from Legare and Thompson-Leduc (2014) sheds some light on perceived challenges as experienced by health professionals. This review will add to the existing literature by highlighting the priorities as perceived by service users and practitioners in mental health, and explore the similarities and difference between stakeholder groups, thereby allowing for greater understanding of the state of the evidence in this emerging area.

The aims of the review are to provide a synthesis of research in the following areas:

- $\quad$ Research examining service user preferences and attitudes towards SDM, including views of what facilitates and hinders the process 
- $\quad$ Research examining professional groups (e.g. psychiatrists', social workers', CPN's) preferences and attitudes towards SDM, including views of what facilitates and hinders the process.

- A comparison of similarities and difference between professionals' and service users' attitudes and views about SDM.

\section{Method}

This review adopts the principles of a qualitative research synthesis (see Suri, 2011) in that it uses a qualitative, iterative and critical approach to synthesizing the primary research in the field. While the synthesis adopts a thematic qualitative approach, studies adopting quantitative (e.g. questionnaire based), qualitative or mixed methods design were included.

The priority for this synthesis was to adopt a purposeful sampling method to obtain an in-depth understanding of existing findings in the field. A key word search of articles published between 1990 and 2016 was undertaken using PubMed. Titles, abstracts and key words were searched using the terms 'shared decision making' 'mental health' and 'attitudes', with related terms included for each category. See appendix two for further details. To supplement the database search, an additional search of publications from prominent known authors in the areas was undertaken, along with snowballing and footnote searching sampling. For example, reference lists were searched of recent known reviews in the area, along with reference lists of highly cited papers in the field. In addition, other 'grey' literature known to the authors (which hadn't been retrieved in the database search) along with an additional search of publications from prominent known authors in the area. This resulted in an initial list of 458 papers.

During the first stage of screening, duplicates were excluded.

Papers were also excluded if they were 1 ) not directly relevant to the field (e.g. not SDM, not mental health, not adult services), 2) were existing review papers, 3) were not written in English, and 4) conducted in forensic settings, drugs and alcohol, and children's mental health service . Methodological rigour was not an imposed exclusion criteria during the screening process and papers adopting qualitative, quantitative and mixed methods research designs were included.

The second stage of screening involved reading article abstracts to ensure the papers were relevant to the scope of the review. At this stage, a team based approach to coding was adopted and two authors (EK and SS) read abstracts from the list of studies. Papers that did not present findings purporting to service user' or practitioner' views and attitudes were excluded. For example, intervention and evaluation studies reporting change in outcomes without reporting findings about stakeholder views and attitudes, were excluded at this stage.

Following this papers were coded in an iterative process, and organised according to the participant group, or, in other words, whether findings purported to practitioner or service user groups. Coding of themes for each group were informed by the aims of review and divided between two authors, EK (for papers reporting service user views), and SS (for papers reporting practitioner's views). Following the coding process, the authors (EK, SS, JH) agreed on themes, and key differences and similarities between the group findings in a team based approach.

[insert diagram here] 
Figure 1: Flow diagram of search strategy

Following the screening process, 43 papers were selected for inclusion in the review.

\section{Findings}

The table in appendix one provides a comprehensive summary of the 43 papers reviewed, including a summary of the key findings.

\subsection{Service user preferences and attitudes towards SDM}

\subsubsection{Service user general endorsement for SDM}

Mental health service users (MHSUs) value participation in decision making and strongly endorse models such as SDM(De las Cuevas, et al., 2012; Puschner et al., 2013; Adams, Drake and Wolford., 2007; Hamann et al., 2005; Patel and Bakken, 2010; Park et al., 2014). Research suggests that the desire for increased involvement in decision making is at least the same (Hamann et al, 2007) if not greater than in other areas of medicine (Hamann et al., 2005; Patel and Bakken, 2010). Desire for greater participation is also found to be greater for service users who are already dissatisfied with services or sceptical about medication (Hamann et al., 2011).

Although the research suggests that service users want to be more involved in the decision making process, an exploration of what this means for service users in the context of receiving mental health services needs expansion. This lack of clarity about what SDM means for MHSUs has been compounded by the development of an array of measures (mostly questionnaires) emphasising different aspects of SDM (see Perestelo-Perez et al in this issue).

\subsubsection{What does SDM mean to service users?}

A defining feature of SDM for service users appears to be the importance of having a voice, being listened to and being supported to express themselves in encounters with mental health practitioners. Aspects of feeling empowered to take the decision or have accountability and ownership over the final decision are seen as less important.

For example, Mahone et al (2011) in a mixed stakeholder focus group study found that service users supported SDM, and in particular, the value it placed on 'putting consumers at a place where their voice is heard' (ibid; p. 9). Service users valued being respected and listened to, although acknowledged that this was associated at times with raised fears about the increased responsibility this implied.

In another focus group study, Rise et al (2013) found similar nuanced attitudes towards SDM. For service users, reaching decisions together and having a voice was particularly important, however and importantly, adopting an informed model whereby service users are seen to have final say and ownership over the decision was not necessarily preferred. Instead, feeling respected by practitioners for their views and experiences was seen as imperative to feeling involved.

Four studies found that service users' conceptualisations of meaningful involvement map more closely to descriptions of SDM as a model, as compared to other empowering models of 
participation, such as the informed model (De las Cuevas et al., 2012; Hamann et al., 2007; Puschner et al., 2013; Park et al., 2014).

For example, De las Cuevas et al (2012) used the Leeds Attitude to Concordance scale (Raynor et al, 2001) to survey psychiatrists and 449 mental health service users from a community outpatient clinic about their attitudes towards SDM. They found that for service user participants there were notable discrepancies in responses between items. For instance, while $95 \%$ of participants agreed that 'better health would follow from cooperation between psychiatrists and patients', only $25 \%$ of respondents agreed with the statement 'During the psychiatrist-patient consultation, it is the patient's decision that is the most important'. Additionally, Park et al (2014) found that while $85 \%$ preferred to be offered options and to be asked their opinions about mental health treatment, more variability was noted in preferences for obtaining knowledge and making final treatment decisions; $61 \%$ preferred to rely on their providers' knowledge and $64 \%$ preferred their provider to make treatment final decisions. Hamann et al (2007) findings that service users demonstrated preference for being involved, and SDM, but not having a final say over the treatment decision. Interestingly, where service users have shown greater desire for autonomy, this correlates with a greater likelihood of not reaching consensus or agreement in meetings (Bonfils et al., 2014).

The theme that service users don't necessarily value ownership of the final decision also appears in qualitative research, and service users here have been described as preferring a two stage process, which acknowledges that in certain more challenging or complex decision, service users feel comfortable and endorse deferring to the clinician's judgment (Whitley \& Woltmann, 2010).

Finally, in this section, two studies of note found that service users highlight the importance of seeing SDM as a process, as oppose to particular outcome (e.g. achieving consensus). De las Cuevas et al (2013) found that mental health service users have a distinct profile in their views of SDM. Mental health service users were found to emphasise the initial steps in SDM, and this pattern differed from other health care settings. Grim et al (2016) in their focus group study found service users supported Elywn's (2013) three stage model of SDM which suggests distinct phases of 'choice talk', 'option talk' and 'decision talk'. This study, similar to the findings of Tanenbaum (2008) emphasise that SDM should also incorporate aspects of both preparation to meetings and follow up stages. SDM models originating from other health care arenas assume that SDM occurs at the point of having an agreed problem established. For mental health service encounters, the description of problems, diagnosis and experiences of treatment are protracted over potentially long periods of time and are often contested. Therefore, in this context it is important for service users to prepare for the encounters by having more information about the purpose and goals of the meeting. Being able to prepare and consider what particular topics, previous experiences and knowledge the service user would like to both prioritise and communicate about at the meeting is emphasised.

In summary, mental health service users are mostly concerned with SDM in respect of feeling listened to and having a voice and being supported to express themselves in encounters with clinicians. The importance of autonomy, or being empowered to take the final decision and ownership are more nuanced and seen to depend on the nature and complexity of the decision to be made. SDM is seen as a process, and not an outcome.

\subsubsection{Perceived enablers and challenges}

Many service users report that they are struggling to be heard or seen as competent and equal in the encounters (Kaminskiy, Morant, Ramon, 2013; Dahlqvist et al. 2015; Tee et al., 2007; Farelly et 
al., 2015; Eliacin et al., 2015; Velligan et al., 2016) and present numerous ways in which these problems can be overcome, as well as offering suggestions for how to enable service users having a greater voice. We present these enablers and challenges under three broad sub headings: Factors related to the service user; factors related to the practitioner and factors related to the wider cultural system.

\subsubsection{Factors related to the service user}

Service users acknowledge that to enable meaningful conversations and achieve shared decision making, effort and energy is required. This takes the form of cognitively preparing for the meeting (Hamann et al, 2016; Grim et al, 2016) actively seeking information (Tanenbaum, 2008; Hamann et al., 2016;) and giving feedback and being an active participant in ongoing conversations. Key enablers to achieving this were viewing themselves as competent and able to have a voice, with ideas of self-efficacy relevant to consider (De las Cuevas et al., 2014).

In Hamann et al's focus group study, service users emphasise the important of giving feedback and informing practitioners of their view, alongside side the need to be open and honest (Hamann et al., 2016).

A few studies have explicitly explored the information that service users desire. Service users report strongly desiring more information about treatment options and in particular a greater understanding of the cost benefit ratio for decisions concerning medication (Barr et al, 2016). In addition, service users seek information from different sources, including conversations with peers, other professionals, and the internet (Stewart et al., 2010). Tanenbaum (2008) used focus groups, to explore types of information MHSUs value and what role it serves in decision making. Service users expressed a strong desire to know more and sought information from multiple sources including the care team, other professional groups (such as pharmacists), peers and the internet. In general, people valued scientific evidence although participants reflected that this at times created problems in that this sometimes reduced the value of the individual accounts. Most importantly, perhaps, focus group members referred to the importance of individuality and the need to treat it as part of the recovery journey where they are themselves an expert. Grim et al (2016) also found that service users felt it extremely important to prepare for meetings by considering what experiential knowledge to share at the meeting.

Linked to this, a key challenge for service users is challenge of being able to express oneself clearly, as well as process information effectively, when unwell (Kaminskiy, Ramon and Morant, 2013). In addition, feelings of perceived inadequacy and a fear of being judged were linked to how involved service users felt they could be (Dahlqvist et al, 2015). Symptomology, diagnosis and stage of recovery is highlighted as influencing both preferences towards SDM as well as the likelihood that SDM will take place (De las Cuevas et al, 2014; Eliacin et al, 2015; Park et al, 2014; Puschner et al., 2016).

\subsubsection{Factors related to the practitioner}

Trust and the importance of the therapeutic relationship in this process is an important enabler that emerges in much of the published research (e.g. Eliacin et al., 2015; Simon et al., 2007; Davidson et al., 2008; Simpson et al., 2016; Street, 2009; Woltman \& Whitley, 2010; Hamann et al., 2016). This may be especially important during more difficult periods and important mitigating factor against 
difficulties of expressing oneself clearly and not feeling able to be fully involved (Woltman \& Whitley, 2010).

Simon et al (2007) undertook qualitative interviews with people receiving services from their GP for depression. This study highlighted that service users had a number of unmet expectations in their consultations regarding treatment. The first concerned expectations for more time in consultations, and the establishment of a trusting relationship with the GP. The formation of a trusting relationship was seen as very important during more difficult times, when service users reported wanting the doctor to take more of a directive role. Davidson Miller and Flanagan (2008) further highlighted helpful relationships. This study was a synthesis of qualitative narrative interviews with service users, focussing on perspectives regarding the utility of treatment. Personal narratives focussed on the positive and helpful role of relationships for medication management. People discussed the prolonged process of first finding the right doctor or nurse, and then eventually the right medication. This study found that the right prescriber was someone who listened to the person, was willing to try different things and who viewed the medication as more than just a way to reduce symptoms (ibid, p.180). In addition, service users expected the GP to be the main source of balanced information about depression treatment options, yet felt that, in general, they did not receive enough information, especially concerning side effects of proposed medication options.

Service users also discussed fear of stigmatisation surrounding medication use as an important concern. Being able to have conversations with other practitioners, such as nurses, as well as other service users, is seen as a helpful way to gain more information about particular options (such as medication) (Stewart et al., 2010).

Involvement in decision making then is seen as reflecting the ideals of a trusting, open and honest relationship with clinicians where the service user is empowered and treated as an equal in the decision making process. It has indeed been suggested that the mechanism by which the therapeutic relationship positively impacts on recovery outcomes in mental health, is via its influence on increased agency and empowerment in decision making (Street et al., 2009), but also as a way of mitigating the problems of not being able to be fully involved in more difficult situations (Woltman and Whitley, 2010).

\subsubsection{Factors related to the wider context.}

As mentioned above, service users often report their views are seen as less important or less valued than practitioners (Velligan et al., 2016; Dahlqvist et al., 2015). In Dahlqvist et al's (2015) grounded theory analysis of 20 group and individual interviews with mental health service users in Sweden, three themes are presented: Being the underdog, being controlled, and being omitted, when describing challenges in participating in SDM. This points to challenges of the wider context in terms of the culture within mental health services, with for example paternalistic practice and other disciplinary forms of power as both prevalent and an important challenge for achieving SDM in practice. For example, Tees et al (2007) present results from a cooperative inquiry study that explored service user participation in decision making. Themes that emerged as inhibiting participation included stigmatization and paternalistic approaches with an over reliance on diagnosis as a basis for clinical judgments. In another study, interviews were conducted on an inpatient ward to explore service user' experiences of medication management and some participants reported that they hadn't realised that they could initiate a discussion about medication, and thought that this was the doctor's role (Stewart et al., 2010). 
Previous experiences of coercion as well as fear of coercion emerges as substantial challenges in our findings. In Mahone et al's (2011) study, previous experiences of coercive practice and the trauma associated with this, led to future mistrust and a lack of willingness to engage in open and meaningful dialogue with practitioners. On the other hand, previous experiences of involuntary treatment has also been found to be associated with a greater desire for autonomy (Hamann et al., 2005) as well as a greater desire for information (Puschner et al., 2016).In a critical narrative analysis of focus groups with service users in an inpatient setting, Stacey et al (2016) report particular challenges associated with inpatient care. One respondent in this study comments about the particular problems associated with SDM in ward rounds (a format involving a group of practitioners as a panel and service user invited to attFend):

'I think the structure (ward review) is ridiculous and frightening. Because all the people on that end, and there's one chair where we sit, like, you're out in a space on your own. . . You're not in that circle. You're the odd one out in other words.' (ibid: p.39).

According to the authors, service users in this context felt like 'outsiders' as well as being at the bottom of the hierarchy.

Factors enhancing participation include a respectful culture that recognised service users' expertise and communicated belief in individual potential as well as the importance of recognising power issues in these helping relationships was highlighted (Tees et al., 2007).

\subsection{Professionals' preferences and attitudes towards SDM}

\subsubsection{Professionals' general endorsement for SDM}

There is strong consensus from research examining practitioner's views that most mental health professionals (e.g. psychiatrists, social workers, Community Psychiatric Nurses (CPNs)) generally support SDM (Barr et al, 2016; Chong, Aslani, \& Chen, 2013a; De Las Cuevas et al., , n.d.; Hamann et al., 2006; Patel, Bakken, \& Ruland, n.d.; Puschner et al., 2016; Rise et al., 2013; S., 2016; Schauer, Everett, del Vecchio, \& Anderson, 2007).

However endorsement of SDM is often seen as situation or topic dependent and overall professionals are seen to have ambivalent attitudes towards SDM (Seale, Chaplin, Lelliott, \& Quirk, 2006). While some research purports that professionals consider SDM to be partially non beneficial under certain circumstances (Hamann et al., 2009), others suggest that it should be condition or decision topic dependent (Chong et al., 2013a; Hamann et al., 2009; Simmons, Hetrick, \& Jorm, n.d.) and some again argue that it is important to not uncritically apply concepts of SDM coming from other areas of health care to MH (Kaminskiy, E., Ramon, S., Morant, 2013). Finally, there is growing literature, suggesting that an idealized view of SDM is distant from standard psychiatric practice(Angell \& Bolden, 2016).

\subsubsection{What does SDM mean to professionals?}

While most research in the field shows that medical professionals endorse SDM, discrepancies exist with reported practice. During a survey among 352 psychiatrists in Germany in 2007, 44\% reported practicing paternalistic decision-making style, $5 \%$ applied an informed choice model and the majority 
(51\%) were in favour of SDM (Hamann et al., 2009). However, in other research, practitioners' perception of SDM bordered on the informed choice model (where the SU makes his own choice, based on the options presented)(Chong, Aslani, \& Chen, 2013b). Other authors conclude that standard SDM models fail to capture the complex and conflictual process that characterizes some psychiatric consultations (Kaminskiy, Ramon, Morant, 2013). Finally, some professionals are concerned that SDM is a threat to professional responsibility and outcomes (Rise et al., 2013).

Moreover, attitudes towards SDM may depend on the professionals' speciality. Hence, it was found that some medical practitioners (psychiatrists, general practitioners) support a more active participation from SU, than other providers (such as pharmacists, occupational therapists). The latter had a higher focus on appreciating SU's needs in decisions, seeing themselves more as advisors in SU's decision making (Chong et al., 2013b). Likewise Colombo et al. (2002) conducted interviews with different stakeholder groups about different explanatory models of mental disorders to understand the influence of "implicit" models on SDM. Whereas psychiatrists and CPNs clearly supported a medical approach (91\% and 61\%), social workers were in favour of the "social" model (48\%). Although there are many different perspectives possible, there is a medical dominance, possibly resulting "from occupational control over several other conflicting interest groups" (Colombo et al., 2002).

Another view of professionals on SDM is an ethical one. Here Drake and Deegan state that psychiatrists tend to forget the ethical imperative to involve patients in preference-sensitive decisions. They recall patient's maturity and that decisions pertaining to the lives of people who use mental health services are personal and need balanced decision support instead of paternalism (Drake \& Deegan, 2009).

Although most professionals support the model of SDM, they often report that flexibility depending on the clinical situation is important (Shepherd, Shorthouse, \& Gask, 2014). Professionals highlight the need to tailor the style of decision making to the situation and the individual patient (Hamann et al., 2009). Hereby professionals generally consider psychosocial matters (i.e. work therapy, future housing) to be more suitable for SDM than medical or legal decisions (Hamann et al., 2009). In addition, in Hamann's randomized controlled trial, doctors recruited the SDM intervention group patients a week later than patients in the control group, indicating that doctors wanted to treat the most severe symptoms first, before embarking on a trial of SDM (Hamann et al., 2006).

Further aspects of what SDM means to professionals derives from Rise et al. (2013). Here, service users and service providers defined involvement as consisting of three core aspects that enable good collaboration: respect, dialogue and SDM (Rise et al., 2013). Professionals can try to maintain partnerships despite current disagreement with SU in some situations in order to be prepared for future shared decisions (Kaminskiy, E., Ramon, S., Morant, 2013).

Recent research in this area has found support from professionals for modified models of SDM, e.g. the "SDM-PLUS"-model. These adapted models are seen by professionals as a way of making SDM more applicable to mental health settings, and allowing greater flexibility and considerations of the ways of overcoming challenges of implementing SDM in practice (Hamann \& Heres, 2014; Mikesell et al., 2016).

\subsubsection{Perceived enablers and challenges}

Many healthcare providers report numerous challenges towards the implementation of SDM and some offer suggestions for how to overcome these barriers. 


\subsubsection{Factors related to the service user}

There are different views of how professionals see their patients. For instance, they see them as "experts by experience", as patients lacking insight, or as patients with questionable validity of views and questionable experiences et cetera. The different views can lead to ambivalent views of service user involvement in decision making (Kaminskiy, Ramon, and Morant, 2013; Seale et al, 2006).

Patient's characteristics like accepting medication, a high preference for participation, recovering from a first episode of illness, being dissatisfied with previous medication and being well informed were seen as speaking strongly in favour of SDM (Hamann et al., 2009). Information valued as enabling by practitioners could be described as professionally owned knowledge. For example, professionals found that patient's knowledge about their diagnosis and related treatment options is important to participate in the SDM process and can be enhanced by information provision (e.g. psychoeducation). In this context, professionals appreciate the help of families and other caregivers and see their increased involvement and the provision of education as facilitators to SDM (Chong et al., 2013b).

Hamann et al. were the first to demonstrate in a controlled trial (comparing a SDM intervention program with routine care) that SDM is possible and feasible for most acutely ill patients with schizophrenia. However, in this study, there were some patients considered to be too ill by their doctors to participate in SDM and not being capable of "making reasonable decisions" (Hamann et al., 2006). Likewise psychiatrists in another survey determined certain patients (i.e. patients in acute psychotic episode) or decisional situations like hospital admission as generally unsuitable for SDM (Hamann et al., 2006; Hamann et al., 2009). This is subsequently leading to paternalistic acting or even using pressure to patients (Quirk et al.,, 2012). Providing information about adverse side-effects can be a facilitator to a certain degree, but it can as well discourage patients to accept treatment (Seale et al., 2006).

Lack of cognitive capacity and lack of insight was the most important barrier to SDM for professionals and is mentioned by several authors (Chong et al., 2013b; Shepherd et al., 2014). These challenges were associated with concerns about patients being both being able to communicate and weigh up information in a meaningful way and also concerning conflict associated with not accepting being ill (deemed as lacking insight). Mental health emergency situations were seen as special challenges to SDM. Therefore, the possible risks of harm to self or others was another barrier to SDM (Mahone et al., 2011a).

According to Chong et al., practitioners report that social stigma associated with mental illness can negatively influence consumers' attitudes towards shared decision-making. Concerns for professionals centre around how labelling and stigma may mean that patients deny or are less open to discussing their diagnosis. Patients therefore engage less in mental health-related concerns and are negatively influenced towards SDM. Furthermore, aspects like social stigma can be linked to cultural backgrounds or religious beliefs (Chong et al., 2013b).

Difficulties with SDM were also expected in patients with long histories of passivity. Prescribers found that (existing) consumer insight was a significant factor for successful SDM and they would "need to learn, what their (the consumers) capabilities are" (Mahone et al., 2011a). For instance after a diagnosis of $A D$, patients sometimes have the approach to "carry on as usual" and therefore might miss the opportunity to discuss and decide on important medical and social topics(Bronner et al., 2016).

Further challenges for SDM are a (partial) gain of knowledge in consumers using the internet, whereas other patients refer to prescribers as the only source of information (Mahone et al., 2011b). However, 
Mahone et al. see prescriber's view on access to the internet just as an "information-exchange issue" without further discussing whether this development is a facilitator or barrier to SDM.

\subsubsection{Factors related to the practitioner}

First, healthcare providers' own attitudes, motivation, willingness, empathy and ability to engage and to implement SDM is an important factor to enable or to impede SDM in clinical practice. Professionals have to be motivated to inform about treatment side-effects for example. Good communication and interpersonal skills are very important (Ramos Pozon, 2016). Exchange of knowledge, respect and especially for service providers - dialogue are significant requirements for mutual involvement (Rise et al., 2013), whereas lacking listening skills and knowledge of alternative treatment can be challenging (Mahone et al., 2011b). Involving a patient can pay off: professionals, who involved a patient more active in decision-making than the patient stated as desired, could accomplish a higher service user satisfaction (Clarke et al., 2015).

Chong et al. (2013) conducted qualitative interviews with practitioners exploring perceptions of barriers and facilitators of not only SDM, but also, interprofessional collaboration. Mental health specialty providers see better mental health expertise, motivation, empathy and genuine interest among general practitioners and pharmacists as a key to better interprofessional collaboration. They found that the desire and/or interest in participating in the SDM process and providing information on illness (psychoeducation) and treatment to service users is seen as a big facilitator of the SDM process (Chong et al., 2013b; Shepherd et al., 2014).

In Mahone's qualitative interviews with 7 focus groups with different stakeholder groups, barriers anticipated by mental health care providers when introducing SDM included the long tradition of the doctor/diagnosis-driven medical, disease-focused model and moral obligations (Provider: "For example, if $I$ as a case manager am making my consumers aware of this new model, this new way of doing things, it's going to take years....So I think that there's got to be more than just service providers. There has got to be agency awareness") (Mahone et al., 2011a).

Farelly et al. report clinicians' barriers to better engaging patients in their Joint Crisis Planintervention: 1. Ambivalence about care planning. 2. Perceptions that they were "already doing SDM". 3. Concerns regarding the clinical "appropriateness of service users'choices". 4. Limited "availability of service users' choices". They, however, acknowledge that patterns might be changing: "I think this is something pretty new that psychiatrists are coming round to in terms of offering choices and, you know, even when we use, talk about a treatment for that matter, I think we're coming to a stage now where we offer the treatment and leave it to the service users to make up theirs minds" (male, Psychiatrist in an interview) (Farrelly et al., 2016).

Some authors see the need of modifying the process of SDM in challenging surroundings. Type of decision, economic and organizational limits, the degree of illness and the patient's wishes are possible challenges (Chong et al., 2013b; Rise et al., 2013). One reason might be that in SDM, health care providers are not in control to the degree they were earlier (Mahone et al., 2011b).

There is misconception in some psychiatrists about whether they really apply SDM - and therefore they see no need to reconsider their behaviour (Hamann et al., 2014).

Finally, it can be challenging for practitioners to develop new skills to satisfy patient's participation and information needs (Reichhart et al. 2008). 


\subsubsection{Factors related to the wider context}

First, accessibility to services is a basis for SDM. There is evidence that being specialized in mental health (psychiatrists vs. primary care physicians) helps to create more SDM. Barr et al. suggest that this might be due to more time pressures in primary care settings (Barr et al., 2016). Although time constraints are still mentioned as barriers to SDM (Chong et al., 2013b), Hamann et al. showed already in 2006, that SDM intervention programs do not take up more of the doctor's time (Hamann et al., 2006).

Professionals saw obligations to society, legal liabilities related to responsible prescribing, a lack of system support, disconnect between inpatient and outpatient prescribing practice, and the need for commitment for "every part of the mental health clinic" as possible barriers and challenges to SDM (Mahone et al., 2011a).

The availability of tools like care plans or decision aids to support SUs involvement is not yet widely known, and are often not widely available (Chong et al., 2013b). Freely available decision aids are beneficial to SDM and need to be further assessed (Simmons, Hetrick, \& Jorm, 2010). Hamann et al. showed that working with decision aids and other psychoeducational tools is possible for patients with schizophrenia and shows promise (see Zisman Ilani et al in this issue for an indepth review of SDM interventions)

The aspect of interprofessional healthcare teams has been included into SDM in mental health recently. Healthcare providers perceive that interprofessional collaboration might facilitate SDM by addressing time barriers and providing more opportunities for consumers to discuss their medicalrelated concerns. Colombo et al. declare, that "effective care means that patients should be able to work with multi-agency teams by being recognised as part of that team"(Colombo et al., 2002).

However, while an interprofessional team approach is seen positively in general, some providers point to confusing and contradictory recommendations between different practitioners (Chong et al., 2013b). Possible problems or gaps within a multi-disciplinary team need to be bridged by creating a common identity as a team (Colombo et al., 2002).

In general, professionals' experience that legal and administrative health and welfare systems can also be barriers instead of only being supportive. In some management or care service structures, efficiency and results can be more important than the quality of services (Ness, Borg, Semb, \& Karlsson, 2014). Finally, all given circumstances might end up in the increasing literature, suggesting, that an "idealized view (of) SDM is distant from standard psychiatric practice"(Angell \& Bolden, 2016).

\section{Discussion}

The primary research presented in this review included studies that adopted a qualitative, quantitative and mixed method design. Within this heterogeneous sample of studies, numerous outcome measures and methods have been employed (see Perestelo-Perez et al in this issue, for a review of SDM measures) and therefore generalisable conclusions are less applicable. In addition, given the broad and iterative search strategy adopted and diversity of methods, the focus of the review has been to present broad themes emergent from the research, but also highlight where debate and lack of consensus emerges. We think that a particularly useful way of exploring these debates is by 
presenting the apparent similarities and differences in views toward SDM between professionals and service users.

\subsection{Similarities and differences between professionals and service users attitudes and views about SDM}

\section{Similarities}

From our findings, service users and practitioners alike endorse shared decision making. Both groups see it as an ethical imperative for mental health services and view the defining features of joint deliberation open dialogue and consideration of the pros and cons of decisions as an important and established part of good mental health practice. Both groups acknowledge that SDM is important for effective care.

However, both service users and practitioners acknowledge that SDM is context dependent, and that the level of involvement will vary from meeting to meeting. Both groups stress that SDM should be viewed as a longer term and dynamic process, as oppose to a particular outcome or list of requirements that need to be performed in a meeting. There is acknowledgement by both service users and practitioners that, at times, the doctor does (and should) take the lead (e.g. De Las Cuevas et al, 2014; Levinson et al, nd).

Linked to this there is acknowledgement that at times it is difficult to fully involve service users or fully weigh up the information on the pros and cons of a particular option. In particular, during periods or situations of lower competence or wellness, meaningful involvement is seen as less practical or realistic. Furthermore, Hamann et al. showed that some patients when acutely ill, they were just not interested in SDM (Hamann et al., 2006).

The administrative and legal framework, other cultural features of mental health services, and stigma is seen as impacting how realistic or achievable meaningful involvement is, and service users and practitioners alike report a gap between idealised models of SDM and actual day to day practice. Importantly, both groups report that in certain circumstances or settings, SDM is far less likely. For practitioners, SDM is not seen as viable for emergency situations and hospital admission, and for service users, ward round meetings are highlighted as a particularly difficult setting (e.g. Hamann et al, 2006;2009; Stacey et al 2016; Mahone et al., 2015).

Both practitioners and service users also highlight the importance of adopting an interdisciplinary approach (e.g. see Chong et al, 2013). Service users value discussing options and seek information from multiple sources, including other members of an interdisciplinary team, and practitioners also acknowledge the importance of interprofessional collaboration as a particular enabler for SDM in mental health.

\section{Differences}

While both practitioners and service users highlight the need to be flexible in implementing shared decision making, and acknowledge that, at certain times, a full weighing up of options is not always preferred or possible, there are some observed differences in the reasons given for reducing 
involvement, and how these 'more difficult' situations are conceptualised by different stakeholders. Practitioners often report particular difficulties involving people who are deemed to lack insight into their difficulties, however, this a problematic area, potentially suffused with how power and authority is enacted in this context. For practitioners, issues of capacity to be involved are a key factor to consider when deciding whether to involve the service user in the decision being taken (e.g. see Chong et al, 2013). This is less apparent in research exploring service user views who acknowledge difficulties in being able to fully participate during periods of being unwell (e.g. difficulties absorbing and processing information the options presented, as well as finding it more difficult to adequately express oneself coherently) (e.g. De Las Cuevas et al, 2014; Kaminskiy, Morant and Ramon, 2013). This observed different also relates to the different emphasis placed on SDM by service users and practitioners. Professionals discuss SDM in terms of adequately preparing for a discussion of treatment options, and see educational programmes and decision aids as potentially helpful in this regard. Service users, on the other hand, highlight SDM as a process, whereby their values, preferences and previous experiences of treatment options, and what is deemed helpful, is more central in conversations with practitioners.

Another observed difference in our findings for our section 'factors associated with the service user' is the priority placed on how and what information is shared in encounters. While both service users and practitioners highlight the need for information about the efficacy and proposed benefits of the option presented, service users also strongly desire to have more information about potential adverse effects (especially concerning decisions surrounding medication).This is potentially compounded by terminology and language used by pharmaceutical industry. Practitioners appear to place less emphasis on the need to present adverse effects, and this is in part appears to be related to fears of possible non-adherence, if a full disclosure of adverse effects takes place. (e.g. Barr et al, 2016)

When discussing factors associated with the practitioner, the importance of the therapeutic relationship as a key facilitator to achieving SDM also receives differing attention among service users' and practitioners' perspectives. Service users (and non-medical professionals) refer to the importance of listening and being listened to, feelings of 'having a voice' and being supported etc. For medical practitioners, while being able to have open and honest dialogue is seen as very important, involvement, as a way of giving service users increased control or ownership over the decision, is more valued. (e.g. Rise et al, 2013). For medical providers' experience, expertise and motivation to implement SDM are seen as important considerations. In addition, practitioners report challenges surrounding the competing priorities of the role, and in particular, the challenges of being accountable and responsible for managing risk (e.g. Mahone et al, n.d). For some these challenges result in concerns regarding the clinical appropriateness of allowing service user choice.

For factors associated with the wider context both groups practitioners also report particular difficulties associated with lack of time and this continues to be a common view presented by practitioners. This is despite Hamann et al's (Hamann et al., 2006) and Loh et al's (2007) research which found that SDM is - in the long run - no more time consuming than normal practice. Time is not highlighted by service users as a particular concern, but instead the problems are of feeling like their views are not respected and feeling marginalised.

Labelling and stigma are seen as a challenge by both practitioners and service users. However, the problems that stigma presents for SDM are seen differently by practitioners and service users. For service users the problems centre on the experience of receiving a psychiatric diagnosis label, and being seen as having less of a voice or being less respected and valued and not being heard, as a consequence of that label. For practitioners, stigma is seen as a problem in that it might prevent a 
person from wanting to accept a diagnosis, possibly leading to lower acceptance, and reduced likelihood of SDM (Chong et al., 2013b). This potentially presents the greatest challenge for SDM in that it highlights the dominating role of a bio medical model for practitioners within mental health services, a system which is organised and underpinned by diagnostic classification systems. This, in turn, presents challenges for service users being able to present their expertise in a meaningful way in encounters.

\subsection{Summary and recommendations}

We have presented themes emergent from research that explores the views and perceptions of SDM for practitioners and service users. In discussing the findings, we have attempted to provide a comparison of the similarities and differences between the stakeholder groups.

While both groups endorse SDM, and acknowledge the need to be flexible with how and when SDM is implemented, there are significant differences in what service users and practitioners describe as preventing or enabling SDM. The differences highlighted point towards different challenges and priorities in SDM for service users and practitioners. In order to encourage greater implementation in every day practice, more time and effort to building a greater joint consensus on what aspects of SDM to prioritise, and how to facilitate its process, is needed. The process of establishing more common ground could itself, be seen as a fruitful way of encouraging greater dialogue, respect and open and honest communication between practitioners and service users. For practitioners, in particular, while SDM is viewed as an ethical imperative, there is a need for greater awareness of the essential contribution of people who use mental health service to knowledge necessary for SDM.

Our findings also point to the need to have separate interventions targeting service users and practitioner group needs (see Zisman-llani et al in this issue for a systematic review of existing SDM interventions in mental health). Priorities for medical providers appear to highlight that being able to 'easily' involve service users as part of everyday is important, and therefore the use of structured training tools, as well as decision aids, may be efficient and helpful in this regard. Additionally, it may be that incentives along with psychoeducational approaches would assist the implementation of SDM. Priorities for service users lie in establishing rapport and trust with practitioners, and feeling empowered to voice experiences and knowledge in meetings. Recovery colleges, and peer support workers offering additional information and further support to embark on SDM may be a useful avenue to explore. Labelling and stigma is perceived as a particular challenge in being able to voice experiences in an open manner. Thus our findings point towards the continued need for an emphasis on the de stigmatisation of mental health. Further research involving patient-doctor dyads and qualitative research exploring views, experiences and perspectives of SDM for service users and practitioners in different settings, would further enhance the knowledge base in this area.

The limitations of this paper should be highlighted. We deliberately chose an inclusive review process and purposefully did not exclude papers on methodological grounds. We believe that for this review a qualitative synthesis was best suited to the enquiry. However, by not adopting the rigour of a full systematic review, it may be that findings were not reported, and that the authors own position in relation to the topic emerges in the presentation of findings. The authors themselves represent different respective positions on this topic (EK, a critical psychology researcher with interest in participatory research in $\mathrm{MH}$, and $\mathrm{SS}$ and $\mathrm{JH}$, practising psychiatrists, and active researchers in SDM in $\mathrm{MH}$ ) and we feel this diversity improves the credibility of the analysis.

Finally, it should be acknowledged that by presenting views of service users' and professionals' views as separate, a rather crude distinction between service users and professionals is drawn in the 
presentation of findings. This has the problem of suggesting that each group is homogenous, or, in other words, that professionals have one position on this topic, and service users another. This was not the intention of this paper. Instead, we aimed to explore the emergent and diverse discourses on this, ultimately, contested, and challenging, issue of rebalancing power in the sharing of decisions in mental health services, and acknowledge that inter as well as intra group differences in discourse are important to highlighting the attitudes and views towards SDM in $\mathrm{MH}$. 


\section{References}

Angell, B., \& Bolden, G. B. (2016). Justifying medication decisions in mental health care: Psychiatrists' accounts for treatment recommendations. http://doi.org/10.1016/j.socscimed.2015.04.029

Barr, P. J., Forcino, R. C., Mishra, M., Blitzer, R., \& Elwyn, G. (2016.). Competing priorities in treatment decision-making: a US national survey of individuals with depression and clinicians who treat depression Setting: Online cross-sectional surveys fielded in. http://doi.org/10.1136/bmjopen-2015-009585

Bronner, K., Perneczky, R., McCabe, R., Kurz, A., \& Hamann, J. (2016). Which medical and social decision topics are important after early diagnosis of Alzheimer's Disease from the perspectives of people with Alzheimer's Disease, spouses and professionals? BMC Research Notes, 9(1), 149. http://doi.org/10.1186/s13104-016-1960-3

Chong, W. W., Aslani, P., \& Chen, T. F. (2013a). Multiple perspectives on shared decision-making and interprofessional collaboration in mental healthcare. Journal of Interprofessional Care, 27(3), 223-30. http://doi.org/10.3109/13561820.2013.767225

Chong, W. W., Aslani, P., \& Chen, T. F. (2013b). Shared decision-making and interprofessional collaboration in mental healthcare: a qualitative study exploring perceptions of barriers and facilitators. Journal of Interprofessional Care, 27(5), 373-9.

http://doi.org/10.3109/13561820.2013.785503

Clarke, E., Puschner, B., Jordan, H., Williams, P., Konrad, J., Kawohl, W., ... Slade, M. (2015). Empowerment and satisfaction in a multinational study of routine clinical practice. Acta Psychiatrica Scandinavica, 131(5), 369-378. http://doi.org/10.1111/acps.12365

Colombo, A., Bendelow, G., Fulford, B., \& Williams, S. (2002). Evaluating the influence of implicit models of mental disorder on process of shared decision making within community-based multi-disciplinary teams. Social Science and Medicine, 56, 1557-1570.

Coulter, A., Parsons, S., \& Askham, J. (2008). Where are the patients in decision-making about their own care. World Health Organization.

Dahlqvist Jönsson P, Schön UK, Rosenberg D, Sandlund M, Svedberg P. Service users' experiences of participation in decision making in mental health services. J Psychiatr Ment Health Nurs. 2015 Nov;22(9):688-97.

De Las Cuevas, C., Rivero-Santana, A., Perestelo-Pérez, L., Pérez-Ramos, J., \& Serrano-Aguilar, P. (n.d.). Attitudes toward concordance in psychiatry: a comparative, cross-sectional study of psychiatric patients and mental health professionals.

De las Cuevas C, Peñate W. (2014) To what extent psychiatric patients feel involved in decision making about their mental health care? Relationships with socio-demographic, clinical, and psychological variables. Acta Neuropsychiatr. Dec;26(6):372-81.

De las Cuevas C, Rivero-Santana A, Perestelo-Pérez L, Pérez-Ramos J, Serrano-Aguilar P. (2012) Attitudes toward concordance in psychiatry: a comparative, cross-sectional study of psychiatric patients and mental health professionals. BMC Psychiatry. May 30;12:53

Drake, R. E., \& Deegan, P. E. (2009). Shared Decision Making Is an Ethical Imperative. Psychiatric Services, 60(8), 1007. http://doi.org/10.1176/appi.ps.60.8.1007

Eliacin J, Salyers MP, Kukla M, Matthias MS. Factors influencing patients' preferences and perceived involvement in shared decision-making in mental health care. J Ment Health. 2015 Feb;24(1):24-8. 
Eliacin J, Salyers MP, Kukla M, Matthias MS. Patients' understanding of shared decision making in a mental health setting. Qual Health Res. 2015

Farrelly, S., Lester, H., Rose, D., Birchwood, M., Marshall, M., Waheed, W., ... Thornicroft, G. (2016). Barriers to shared decision making in mental health care: qualitative study of the Joint Crisis Plan for psychosis. Health Expectations, 19(2), 448-458. http://doi.org/10.1111/hex.12368

Gonzalez-Lorenzo M, Serrano-Aguilar P, Sanz EJ. Mental health professionals' attitudes to partnership in medicine taking: a validation study of the Leeds Attitude to Concordance Scale II. Pharmacoepidemiol Drug Saf. 2012 Feb;21(2):123-9.

Grim K, Rosenberg D, Svedberg P, Schön UK. Shared decision-making in mental health care-A user perspective on decisional needs in community-based services. Int J Qual Stud Health Wellbeing. 2016 May 9;11:30563. doi:

Hamann, J., \& Heres, S. (2014). Adapting shared decision making for individuals with severe mental illness. Psychiatr Serv, 65(12), 1483-1486. http://doi.org/10.1176/appi.ps. 201400307

Hamann, J., Langer, B., Winkler, V., Busch, R., Cohen, R., Leucht, S., \& Kissling, W. (2006). Shared decision making for in-patients with schizophrenia. Acta Psychiatrica Scandinavica, 114(4), 265-273. http://doi.org/10.1111/j.1600-0447.2006.00798.x

Hamann, J., Maris, N., losifidou, P., Mendel, R., Cohen, R., Wolf, P., \& Kissling, W. (2014). Effects of a question prompt sheet on active patient behaviour: a randomized controlled trial with depressed outpatients. The International Journal of Social Psychiatry, 60(3), 227-35. http://doi.org/10.1177/0020764013482311

Hamann, J., Mendel, R., Cohen, R., Heres, S., Ziegler, M., Bühner, M., \& Kissling, W. (2009). Psychiatrists' use of shared decision making in the treatment of schizophrenia: patient characteristics and decision topics. Psychiatric Services (Washington, D.C.), 60(8), 1107-12. http://doi.org/10.1176/appi.ps.60.8.1107

Kaminskiy, E. (2015). The Elephant in the Room: A Theoretical Examination of Power for Shared Decision Making in Psychiatric Medication Management. Intersectionalities: A Global Journal of Social Work Analysis, Research, Polity, and Practice, 4(2), 19-38.

Kaminskiy, E., Ramon, S., Morant, N. (2013). Exploring Shared Decision Making for Psychiatric Medication Management.

Légaré, F., Ratté, S., Gravel, K. and Graham, I.D. (2008). Barriers and facilitators to implementing shared decision-making in clinical practice: update of a systematic review of health professionals' perceptions. Patient education and counseling, 73(3), pp.526-535.

Légaré, F. and Thompson-Leduc, P. (2014.) Twelve myths about shared decision making. Patient education and counseling, 96(3), pp.281-286.

Mahone, I. H., Farrell, S., Hinton, I., Johnson, R., Moody, D., Rifkin, K., ... Barker, M. R. (2011a). Shared Decision Making in Mental Health Treatment: Qualitative Findings from Stakeholder Focus Groups. Cell, Fax, 434-295. http://doi.org/10.1016/j.apnu.2011.04.003

Mahone, I. H., Farrell, S., Hinton, I., Johnson, R., Moody, D., Rifkin, K., ... Barker, M. R. (2011b). Shared Decision Making in Mental Health Treatment: Qualitative Findings From Stakeholder Focus Groups. Archives of Psychiatric Nursing, 25(6), e27-e36.

http://doi.org/10.1016/j.apnu.2011.04.003

Mikesell, L., Bromley, E., Young, A. S., Vona, P., \& Zima, B. (2016). Integrating Client and Clinician Perspectives on Psychotropic Medication Decisions: Developing a Communication-Centered 
Epistemic Model of Shared Decision Making for Mental Health Contexts. Health Communication, 31(6), 707-17. http://doi.org/10.1080/10410236.2014.993296

Miyamoto $\mathrm{Y}$, Hashimoto-Koichi R, Akiyama M, Takamura S. Mental health and social service needs for mental health service users in Japan: a cross-sectional survey of client- and staff-perceived needs. Int J Ment Health Syst. 2015 Apr 10;9:19. doi: 10.1186/s13033-015-0009-7. eCollection 2015.

Moran GS, Baruch Y, Azaiza F, Lachman M. Why do mental health consumers who receive rehabilitation services, are not using them? A Qualitative Investigationof Users' Perspectives in Israel. Community Ment Health J. 2015 Jul 23. [Epub ahead of print] PubMed PMID: 26202546.

Ness, O., Borg, M., Semb, R., \& Karlsson, B. (2014). "Walking alongside:" collaborative practices in mental health and substance use care. International Journal of Mental Health Systems, 8(1), 55. http://doi.org/10.1186/1752-4458-8-55

Park, S. G., Derman, M., Dixon, L. B., Brown, C. H., Klingaman, E. A., Fang, L. J., ... \& Kreyenbuhl, J. (2014). Factors Associated With Shared Decision-Making Preferences Among Veterans With Serious Mental Illness. Psychiatric Services.

Patel, S. R., Bakken, S., \& Ruland, C. (n.d.). Recent Advances in Shared Decision Making for Mental Health. http://doi.org/10.1097/YCO.0b013e32830eb6b4

Pozon, S. R. (2016). Shared decision making in mental health : Myths, barriers, mitos, barreras y

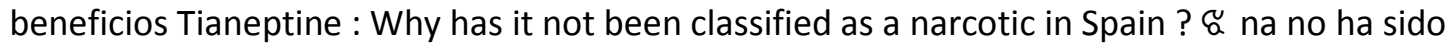
Tianeptina : ¿ por qué en Espa . Rev Psiquiatr Salud Ment, 8-9.

Puschner, B., Becker, T., Mayer, B., Jordan, H., Maj, M., Fiorillo, A., ... Slade, M. (2016). Clinical decision making and outcome in the routine care of people with severe mental illness across Europe (CEDAR). Epidemiology and Psychiatric Sciences, 25(01), 69-79. http://doi.org/10.1017/

A. Quirk (2014) Obstacles to Shared Decision Making in Psychiatric Practice. Lambert Academic Publishing: Germany

Quirk, A., Chaplin, R., Lelliott, P., \& Seale, C. (2012). How pressure is applied in shared decisions about antipsychotic medication: A conversation analytic study of psychiatric outpatient consultations. Sociology of Health and IIIness, 34(1), 95-113. http://doi.org/10.1111/j.14679566.2011.01363.x

Reichhart, T., Kissling, W., Scheuring, E., \& Hamann, J. (2008). Patient participation in german psychiatry - a critical review. Psychiatrische Praxis, 35(3), 111-21. http://doi.org/10.1055/s2007-986410

Rise, M. B., Solbjr, M., Lara, M. C., Westerlund, H., Grimstad, H., \& Steinsbekk, A. (2013). Same description, different values. How service users and providers define patient and public involvement in health care. Health Expectations, 16(3), 266-276. http://doi.org/10.1111/j.1369-7625.2011.00713.x

Schauer, C., Everett, A., del Vecchio, P., \& Anderson, L. (2007). Promoting the value and practice of shared decision-making in mental health care. Psychiatric Rehabilitation Journal, 31(1), 54-61. Retrieved from http://www.ncbi.nlm.nih.gov/pubmed/17694716

Seale, C., Chaplin, R., Lelliott, P., \& Quirk, A. (2006). Sharing decisions in consultations involving antipsychotic medication: A qualitative study of psychiatrists' experiences. Social Science and Medicine, 62(11), 2861-2873. http://doi.org/10.1016/j.socscimed.2005.11.002

Shepherd, A., Shorthouse, O., \& Gask, L. (2014). Consultant psychiatrists' experiences of and 
attitudes towards shared decision making in antipsychotic prescribing, a qualitative study. $B M C$ Psychiatry, 14(1), 127. http://doi.org/10.1186/1471-244X-14-127

Simmons, M. B., Hetrick, S. E., \& Jorm, A. F. (n.d.). Making decisions about treatment for young people diagnosed with depressive disorders: a qualitative study of clinicians' experiences.

Simmons, M., Hetrick, S., \& Jorm, A. (2010). Shared decision-making: benefits, barriers and current opportunities for application. Australasian Psychiatry: Bulletin of Royal Australian and New Zealand College of Psychiatrists, 18(5), 394-7. http://doi.org/10.3109/10398562.2010.499944

Simpson A, Hannigan B, Coffey M, Barlow S, Cohen R, Jones A, Všetečková J, Faulkner A, Thornton A, Cartwright $M$. Recovery-focused care planning and coordination in England and Wales: a crossnational mixed methods comparative case study. BMC Psychiatry. 2016 May 16;16(1):

Stacey, G., Felton, A., Morgan, A., Stickley, T., Willis, M., Diamond, B., Houghton, P., Johnson, B. and Dumenya, J., 2016. A critical narrative analysis of shared decision-making in acute inpatient mental health care. Journal of interprofessional care, 30(1), pp.35-41.

Stewart, D. C., Anthony, G. B., \& Chesson, R. (2010). 'It's not my job. I'm the patient not the doctor': Patient perspectives on medicines management in the treatment of schizophrenia. Patient education and counseling, 78(2), 212-217.

Suri, H., 2011. Purposeful sampling in qualitative research synthesis. Qualitative Research Journal, 11(2), pp.63-75.

Velligan DI, Roberts DL, Sierra C, Fredrick MM, Roach MJ. What Patients With Severe Mental Illness Transitioning From Hospital to Community Have to Say About Care and Shared DecisionMaking. Issues Ment Health Nurs. 2016 Apr 7:1-6. [Epub ahead of print] PubMed PMID: 27054268.

Woltmann E.M. \& Whitley R. (2010) Shared decision making in public mental health care: perspectives from consumers living with severe mental illness. Psychiatr Rehabil J. Summer;34(1):29-36. 